\title{
El Comercio justo de Ecuador a España, Quito, Ecuador, Abril del 2009
}

\section{Autores:}

Exp. César Acosta Briones

Eco. César Marcillo Vaca 


\section{Resumen}

El proyecto de: "El Comercio Justo de Ecuador a España”, es una nueva alternativa de comercialización de productos, para mejorar las condiciones de vida de los menos favorecidos, del Ecuador, creando organizaciones con producciones que agreguen valor para el mercado doméstico e internacional. El proyecto responde a la realidad en que viven los estratos económicos más bajos del país, y a la necesidad de buscar respuestas que mejoren la situación en forma inmediata y mediata de la población. Para este propósito, se promueve una empresa nacional de comercialización de Comercio Justo que permita dinamizar el modelo organizado por la UTE. En el estudio se aplicó un modelo deductivo-inductivo. Se efectuó un análisis del mercado Interno y Externo, que permitió identificar el incremento en el volumen de ventas, involucramiento de nuevos actores en el Comercio Justo, a nivel nacional e internacional. Alcanzando cifras de exportaciones totales en el 2007, de $\$ 25$ millones que son alentadoras y justifican el apoyo que podría dar el Estado a éste proceso. El proyecto permitió cumplir con la oferta de los diez productos que constituyeron la propuesta: Comercio Justo entre Ecuador y España; Manual de Comercio Justo; Economía solidaria; Marketing Social; Emprendimiento; Prospectiva; Evento; Convenio con la Universidad de Mondragón (España); Sello FLO; Empresa de Comercialización. La investigación hizo posible llegar a las siguientes conclusiones: La modalidad de comercio Justo para comercializar productos al mercado mundial ha permitido el ingreso de divisas por 25 millones de dólares en el 2007. No hay apoyo oficial y académico en el Ecuador para impulsar esta nueva forma de comercio. A nivel académico la terminología sobre: Comercio Justo, Economía Solidaria, Finanzas Justas, Emprendimientos solidarios, es desconocida. El consumidor nacional desconoce esta nueva alternativa de comercio justo, en el Ecuador y los beneficios que esta podría representar para las personas de escasos ingresos. Existen organizaciones privadas, en el país, que desde hace algunos años están trabajando en este esquema como: Sinchi Sacha, Maquita Cushunchic, Camari, entre otras. Sería pertinente la incorporación a la malla curricular universitaria materias relacionadas con: Comercio Justo, Economía Solidaria, Finanzas Justas, Emprendimientos Solidarios, etc. La Facultad de Ingeniería de Empresas y Negocios de la UTE podría tener una titulación en Economía Solidaría y Comercio Justo. El convenio macro con la Universidad de Mondragón (España), podría ser importante y valioso para aprovechar la información y conocimientos en materia de cooperativas productivas y sus resultados exitosos.

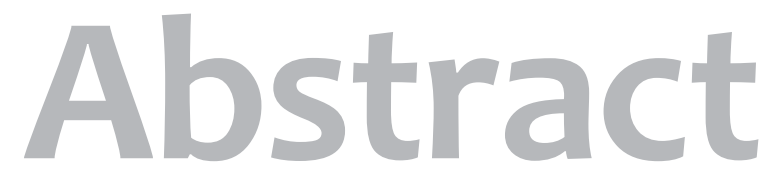

The "Fair Trade between Ecuador and Spain" project is a new option for commercializing products by creating enterprises where value is added in a household and international market in order to improve the life conditions of the poorest population of Ecuador. The project responds to the need of finding immediate and mediate solutions that may help improve the reality in which poor people of the country live. To achieve this goal, it is promoted a national Fair Trade enterprise that allows putting in practice the model proposed by UTE. Within the study, an inductive and deductive model was applied together with an internal and external market research. These procedures allowed identifying the increasing sale volume and new actors of the Fair Trade to a national and international level. The result was an encouraging total of \$25 millions in the year 2007; therefore, the Government's support to the project would be justified. The project fulfilled the offer of ten products that were part of the initial proposal: Fair Trade between Ecuador and Spain; Fair Trade Manual; Joint Economy; Social Marketing; Enterprising; Prospecting; Event; Alliance with the Mondragon University (Spain); 
FLO seal; Trading Enterprise. After the research the following conclusions were thrown: The model of Fair Trade to commercialize products worldwide has allowed an income of \$25 millions in 2007. In Ecuador there is neither official nor academic support to promote this new way of trading. The meaning of: Fair Trade, Joint Economy, Fair Finances, Joint Enterprises is still unknown at an academic level. Neither does the Ecuadorian consumer know about this Fair Trade option nor about the potential benefits that poor people might obtain from it. There are private institutions in the country working for years under this scheme such as: Sinchi Sacha, Maquita Cushunchic, Camari, among others. It would be appropriate to include in the University academic syllabi subjects related to Fair Trade, Joint Economy or Fair Finances. The Business school would offer majors in some of the mentioned topics at UTE. The macro deal with the Mondragon University (Spain) could be an important and valuable source of information and knowledge concerning subjects about productive financial institutions and their successful results.

\section{Introducción}

“El Comercio Justo", Para mejorar las condiciones de vida de los menos favorecidos está ganando terrero en el mercado mundial, la autogestión para crear organizaciones con producciones que agreguen valor para distribuir en los mercados domésticos y los internacionales. Organismos como el Banco Mundial, el Banco Interamericano de Desarrollo y ONG's impulsan micro emprendimientos, las micro finanzas para solucionar los problemas señalados.

Para 1969, se advierte en Europa un interés por adquirir productos de las organizaciones comunitarias pobres de los países del Sur y, el movimiento de Comercio Justo, que en principio, era un pequeño círculo comienza a expandirse, a pesar de no contar con el apoyo de los gobiernos, centros académicos, salvo raras excepciones.

\section{- Objetivo}

Analizar el incremento de las exportaciones mediante Comercio Justo por el efecto económico social que estas representan en relación con las ventas externas tradicionales.

Pues un dólar exportado en artesanía o café orgánico, tiene un efecto económico y social distributivo impresionante. Frente a un dólar exportado proveniente del petróleo por ejemplo o banano de las grandes empresas, las utilidades se reparten equitativamente en el primer caso o en el segundo no llegan a los que deberían ser sus potenciales beneficiarios.

\section{Materiales, Métodos y Procedimientos}

\section{- Diseño de la Investigación}

La investigación se orientó a proponer una nueva posibilidad de comercialización internacional basada en las producciones con valores de las comunidades más relegadas y en su mayoría rurales del Ecuador, para orientarlas al mercado más idóneo de Europa: España.

El Comercio Justo, como se identifica esta modalidad, es parte de la Economía Solidaria, que involucra el combate a la pobreza y la exclusión. Actualmente, bajo éste sistema se registran US\$ 25 millones anuales de exportación ecuatoriana hacia diferentes países, europeos de preferencia a España.

\section{- Métodos generales y específicos de investigación}

La metodología que utilizó en el proyecto fue el método deductivo- inductivo, es decir, partir de lo general a lo particular. Para conocer primero las realidades del Comercio Justo de los países 
involucrados, y luego particularizar cada componente del Comercio Justo, hasta la elaboración del proyecto específico de crear una empresa nacional comercializadora de Comercio Justo, en el Ecuador auspiciada por la UTE.

Para este propósito, se recopiló información primaria y secundaria. La información primaria se utilizó en el método inductivo partiendo de las informaciones obtenidas en los sectores productivos y luego infiriendo a una realidad nacional, mediante investigación realizada en visitas de campo, a las principales organizaciones de las localidades de la provincia de Imbabura y de la provincia de Pichincha.

El trabajo se complementó con reuniones de trabajo con ONGs que están exportando bajo el Comercio Justo como: Camari, Maquita Cusumchi, Shichi Sacha, entre otras. También se concretaron con participaciones como proveedores de información: El Consejo Provincial de Pichincha sobre Comercio Justo en esta provincia, el Instituto del Eco desarrollo de la Amazonía ECORADE sobre las provincias de ésta región, y el Ministerio de Industrias en el temas de emprendimiento, Ministerio de Inclusión Económica y Social, CORPEI por el sector privado. Instituciones que trabajan por mandato legal en las actividades productivas con alto contenido social.

En España, se concretó el trabajo con una visita de cinco días a la ciudad de Mondragón, un ejemplo de producción cooperativista y solidaria, así como para conocer el impulso de la academia en la comercialización justa. En la ciudad de Madrid, se estableció contactos con centros de distribución e importación de productos, en la modalidad de Comercio Justo, para cuantificar la demanda, conocer las redes de tiendas solidarias de consumidores, la organización central de migrantes $y$, finalmente, a la entidad certificadora del sello de comercio justo FLO (Fair Trade Labelling Organizations), que abaliza la comercialización en el mercado internacional.
- Técnicas Generales y Específicas de Investigación

\section{Primarias}

- Entrevistas con ejecutivos de las ONGs en Ecuador y España identificadas con el Comercio Justo, Ministerio de Industrias y Competitividad del Ecuador, Ministerio de Inclusión Económica y Social, COPERI, Certificadora del sello FLO, en España.

- Entrevistas con las empresas que están dedicadas a la comercialización de productos en la modalidad de Comercio Justo en el Ecuador.

- Observación a tiendas solidarias de Comercio Justo de Ecuador y España, para visualizar la presentación de los productos, técnicas de comercialización, precios, promoción y publicidad

\section{Secundarias}

- Libros: El malestar en la globalización, Joseph E. Stiglitz, México 2006

- Cómo hacer que funcione la Globalización, Joseph E. Stiglitz, Colombia 2006

- Comercio Justo para todos, Joseph E. Stiglitz, México 2007

- Economía Solidaria, Eco. César Marcillo Vaca, Ecuador 2006

- Diagnóstico de las operaciones de las PYMES, Javier Leopoldo Lozano, México, 2005.

- Ecuador su realidad, Lola Vásquez, Napoleón Saltos, Ecuador, 2008.

1. Revistas: Varios temas de Comercio Justo, Economía 
Solidaria facilitados por organizaciones españolas, durante la visita realizada a España en el marzo del 2008.

2. Internet: Artículos sobre Comercio Justo, Economía solidaria, Finanzas solidarias, Micro emprendimientos.

\section{- Población/ Muestra}

Para alcanzar el objetivo de la investigación, que básicamente se centró en la promoción de una nueva alternativa de comercialización, se promocionó la realización de un Seminario de la Feria y Foro de Comercio Justo, realizado del 7 al 8 de agosto del 2008, en los salones del Consejo Provincial de Pichincha.

Creando un espacio dirigido a to da la sociedad ecuatoriana que quiere nuevas alternativas de organización , producción, comercio y consumo, basados en la solidaridad, la protección ambiental y un modelo de economía donde lo más importante no es el dinero sino el ser humano.

Para promocionar el encuentro y el intercambio solidario entre productores y consumidores, sumando otros actores de los diversos sectores que confluyen en la economía solidaria como las organizaciones miembros del Comercio Justo, finanzas, servicios, comercialización, entidades educativas, tecnológicas, los gobiernos locales y nacionales.

Auspiciada entre otros por la Universidad Tecnológica Equinoccial, con la presencia de 200 asistentes, que representaban al sector oficial, privado, ONG's y académico como: MIC, Ministerio de Inclusión Económica y Social, Consejo Provincial de Pichincha, CONQUITO, CORPEI, Camari, Sichi Sacha Maquita Cushunchic, IFATLatinoamericana, PODER, RELACC, Visión Mundial Ecuador, FEPP y UTE, como organizadores y auspiciantes.

Quienes tuvieron relevante participación y condiciones para resaltar la necesidad de ampliar las oportunidades de micro emprendimientos que contribuyan al mejoramiento de la calidad de vida de la población de menos recursos. A través de un ciclo de ponencias que cubrieron los siguientes temas:

- Trayectoria del Comercio Justo a nivel mundial: Estándares sociales, ecológicos y comerciales.

- Comercio Justo en cifras: situación mundial regional, obstáculos y perspectivas.

- Expectativas de los mercados internacionales en el comercio justo.

- La importancia de las certificaciones en comercio justo.

- El Comercio Justo en el Ecuador: Cifras y perspectivas, relaciones con la economía solidaria en el país y en América Latina.

- Posición del gobierno ecuatoriano frente a la economía solidaria y el comercio justo.

- Los gobiernos seccionales en apoyo a la economía solidaria y comercio justo.

\section{Resultados y Discusión}

Durante el desarrollo del evento se constituyeron siete mesas de trabajo con el siguiente temario:

\section{Día 7 de agosto}

\begin{tabular}{|c|c|}
\hline Mesas de trabajo & Organización líder \\
\hline $\begin{array}{l}\text { 1. La academia como otro actor del } \\
\text { comercio justo }\end{array}$ & Universidad Tecnológica Equinoccial \\
\hline $\begin{array}{l}\text { 2. El comercio justo en las estrategias de } \\
\text { desarrollo económico del sector } \\
\text { público }\end{array}$ & $\begin{array}{l}\text { Consejo Provincial de Pichincha y } \\
\text { CONQUITO }\end{array}$ \\
\hline $\begin{array}{l}\text { 3. Incursión en mercados nacionales e } \\
\text { internacionales de comercio justo }\end{array}$ & CORPEI \\
\hline $\begin{array}{l}\text { Plenaria para exposición y análisis de } \\
\text { conclusiones de las mesas de trabajo }\end{array}$ & RELACC \\
\hline
\end{tabular}


Día 8 de Agosto

\begin{tabular}{|c|c|}
\hline Mesas de Trabajo & Organización líder \\
\hline $\begin{array}{l}\text { 1. Estrategias de apoyo a grupos } \\
\text { campesinos }\end{array}$ & FEPP-CAMARI \\
\hline $\begin{array}{l}\text { 2. Artesanía con identidad o tendencias de } \\
\text { moda }\end{array}$ & Consultor \\
\hline $\begin{array}{l}\text { 3. Turismo solidario como alternativa de } \\
\text { desarrollo comunitario }\end{array}$ & $\mathrm{MCCH}$ \\
\hline 4. La plata del pueblo para el pueblo & FEPP-Codesarrollo \\
\hline $\begin{array}{l}\text { Plenaria para exposición y análisis de las } \\
\text { conclusiones }\end{array}$ & FEPP-Codesarrollo \\
\hline
\end{tabular}

Las exposiciones y mesas de trabajo permitieron crear las condiciones favorables para sentar las bases para impulsar el modelo alternativo del Comercio Justo, como una importante alternativa de emprendimiento y comercialización, que podrá mejorar las condiciones de vida de las clases menos favorecidas del país.

\section{Cuadro No. 1}

Ventas del comercio justo en países de Europa. 2005

\begin{tabular}{|l|r|}
\hline \multicolumn{1}{|c|}{ Países europeos } & \multicolumn{1}{c|}{$\begin{array}{r}\text { Ventas Totales } \\
\text { Euros }\end{array}$} \\
\hline Alemania & 133.035 .000 \\
\hline Austria & 36.046 .000 \\
\hline Bélgica & 44.790 .000 \\
\hline Dinamarca & 13.062 .000 \\
\hline Finlandia & 8.794 .000 \\
\hline Francia & 87.774 .000 \\
\hline Holanda & 85.983 .000 \\
\hline Irlanda & 6.325 .000 \\
\hline Italia & 61.165 .000 \\
\hline Luxemburgo & 2.800 .000 \\
\hline Noruega & 4.540 .000 \\
\hline Portugal & 386.000 \\
\hline Reino Unido & 276.246 .000 \\
\hline Suecia & 8.396 .000 \\
\hline Suiza & 138.023 .655 \\
\hline España & 20.159 .000 \\
\hline TOTAL & 927.524 .655 \\
\hline
\end{tabular}

Fuente: SETEM a partir de los datos de EFTA

Elaboración: UTE
A estos 927 millones de euros de las transacciones europeas se suman las de Estados Unidos, Japón y Canadá y se estima que en dicho año la cifra se situó en los 1.100 millones de euros.

Entre los años 2000 al 2005, la tasa de crecimiento del comercio justo, según SETEM, registró el 20\% anual, pero en el 2006, la variación se eleva a 42\% y para el 2007 el nivel está en 47\%. Es fácil deducir que, por ello, los ojos de las grandes empresas se ponen en estos comportamientos con mucha atención, aún a pesar que su peso relativo en el comercio mundial es todavía muy insignificante.

Los productos del comercio justo son numerosos, pero el más importante es el café, y los alimentos desplazaron a las artesanías, en los últimos años se están incorporando productos muy novedosos como textiles, camisetas, balones de fútbol, licores y, más.

La Fair Trade Labelling Organization, (FLO), señala que hay productos de comercio justo (es decir, aquellos distribuidos por todos sus operadores) en 55.000 supermercados de toda Europa. Esta realidad tiene el rechazo por los defensores del comercio justo puro, los cuales consideran que este comercio alternativo debe ser ejecutado solamente por las tiendas solidarias, según ellos a futuro toda la distribución final la realizarán las transnacionales, desvirtuando la esencia del mismo.

Se ejemplifica en forma relevante a la transnacional suiza Nestlé que elabora un café soluble del comercio justo y le compite a Max Haavelar, que en cambio es un gran representante de este tipo de comercio. El nuevo producto de Nestlé “arábica 100\%” proviene de las plantaciones organizadas en cooperativas de El Salvador y Etiopía.

El gigante suizo manifiesta que está en la actividad como un compromiso de responsabilidad social de la empresa y una forma de ayudar a salir de la miseria a los pequeños productores de café.

En Gran Bretaña, se considera positivo la actitud de 
la transnacional, en tal virtud entregó a la marca "Nescafé Partners'Blend" el sello de "café surgido del comercio justo". En la mancomunidad británica está de moda el «ethical shopping», como se le llama en inglés al comercio justo y el consumo de este nuevo producto controvertido representa el 3\% del mercado total del café soluble. Un mercado en plena expansión.

Las organizaciones defensoras de los derechos humanos critican a la firma Nestlé, y establecen que su participación es un golpe de efecto de mercadotecnia para mejorar su imagen corporativa, pues el objetivo base de la multinacional, denuncian es el enriquecimiento de sus accionistas y no el combate a la pobreza en el mundo. Ellos recuerdan que Nestlé en Colombia, licenció a los sindicalistas de sus fábricas por protestar por las condiciones laborales de los trabajadores de sus plantaciones de café.

\section{- Los productores, productos, exportaciones y exportadores}

del Comercio Justo en Ecuador: cooperativas, asociaciones, microempresas.

En el país se identifican muchos productores de comercio justo, que productores no están certificados, pero que se consideran parte intrínseca de este movimiento.

De la investigación realizada se resaltan los casos más relevantes que ya han obtenido el sello FLO o que pertenecen a organizaciones de carácter mundial y regional. Ecuador forma parte del IFAT, y en la actualidad dirige al IFAT capítulo América Latina, personal de la Fundación Sinchi Sacha.

Porotrolado, EcuadoressededeRELACC, RedLatinoamericana de Comercio Comunitario y la oficina central está en Quito.

Tres organizaciones comercializadoras ecuatorianas como CAMARI, MCCH o Maquita Cushunchic (Comercializando como Hermanos) y Sinchi Sacha, impulsan las ventas del comercio justo desde diferentes ámbitos.

La Fundación CAMARI, cumple más de 30 años con resultados bastante halagadores. MCCH también sobrepasa los 20 años, promueve el turismo comunitario. Sinchi Sacha está más dedicada a vender artesanías en el Ecuador y a promover el turismo.

No existen registros abundantes de la Región Oriental, más bien casos aislados como por ejemplo la producción y exportación del Comité Empresarial Aroma Amazónico que agrupa a 2.000 productores se localiza en Nueva Loja, en el año 2007 exportó 75 toneladas con patente amazónica, sello de comercio justo y la calidad ambiental de Rainforest Alliance. Sus compradores son Yachana Gourmet, Ecuatoriana de Chocolates y Kraft Food de Alemania.

A continuación se detalla a 20 organizaciones que tienen el sello FLO y los grupos de productos a los que pertenecen. Los grupos son de flores y plantas, bananos, frutas secas, quinua, cacao, hierbas medicinales, café y jugos. 


\section{Cuadro No 2}

\section{Ecuador: Listado de Organizaciones con sello FLO}

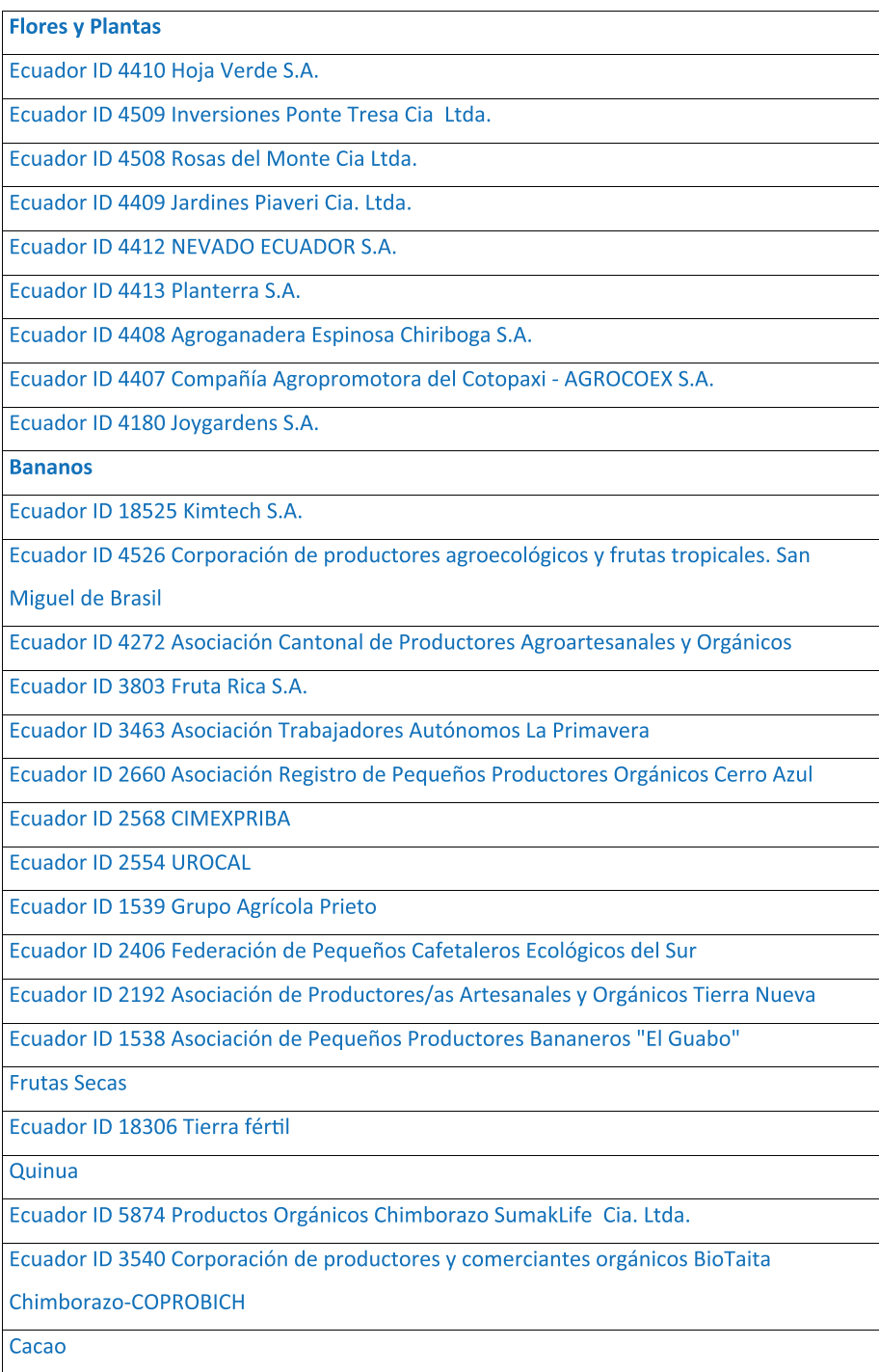

Fuente: FLO Madrid

Elaboración: UTE

\section{- Los Productos}

Información detallada sobre las tendencias del comercio justo desde Ecuador hacia el resto del mundo no está disponible por la falta del apoyo gubernamental para levantar estadísticas sobre este tema.
Los datos que se insertan a continuación corresponden a la información que manejan las tres comercializadoras señaladas: Camari, MCCH y Sinchi Sacha.

Para éstas, el comercio justo en los tres últimos años ha crecido de una manera impresionante así: en el año 2005 el valor exportado era de USD\$16 millones, en el 2006 de USD \$ 25 millones y en el 2007 de USD\$ 30 millones. Un crecimiento mucho más dinámico que el del comercio exterior convencional, excluido el petróleo, ya que éste por el crecimiento de los precios distorsiona cualquier comparación.

Una última información proporcionada por la Fundación FEDA de España, entrega el “Directorio de la Dignidad 2008”, que agrupa a 15 organizaciones ecuatorianas y que exportan una diversidad de productos como se observa en Anexo No 1. Los responsables del Directorio aclaran que constan aquellos productores más idóneos que se apegan los principios del comercio justo y aunque no es lo exhaustivo que se desearía es una base muy importante para conocer la oferta de productos ecuatorianos.

- El Comercio Justo en España, funcionamiento y perspectivas.

- Las Ventas Totales en España período 2000- 2005

Entre los años 2004 y 2005, las ventas totales de productos de Comercio Justo en España registraron la mayor tasa de crecimiento desde que se inició la década, un 28,4\%, lo que permite que la cifra final pase de los cerca de 11,7 millones de euros del 2004 a los casi 15 millones del último año. Este intenso crecimiento compensa la menor tasa de variación del bienio 2003-2004, cuando las ventas aumentaron por debajo del 10\%. Aún así, el valor registrado en 2005 consigue más que duplicar los valores del año 2000 (menos de 7 millones de euros), y confirma la tendencia al crecimiento de una actividad que ha conseguido situar su tasa de aumento promedio 
anual en los seis años analizados en un notable $16,9 \%$.

En España, en el año 2007 los productos de Comercio Justo certificado crecieron al 105\% comparado con el año 2006, lo que demuestra la tendencia creciente, similar a la mundial y a la europea.

Los análisis detallados se circunscriben hasta el año 2005, ya que los de los años 2006 y 2007, SETEM entregará al público para fines del año 2008 o principios del 2009.

\section{- Ventas por Comunidades Autónomas}

Cuando los datos se desagregan por comunidades autónomas se evidencian las distintas dinámicas de crecimiento regional. Así, entre los años 2000 y 2005, sólo cinco comunidades han registrado una tasa media de crecimiento de las ventas superior a la correspondiente al conjunto del territorio español. Estas comunidades se sitúan geográficamente en el centro de la península (Madrid y las dos Castillas), y en los extremos occidental y oriental de la zona norte del país (Galicia y Cataluña, respectivamente).

Estas tasas de crecimiento regional diferenciados provocaron los cambios en la estructura de ventas por Comunidades Autónomas. Efectivamente, en el año 2000, Cataluña, Madrid y Andalucía representaban, en conjunto, la mitad del total de las ventas realizadas en España (50,1\%); si se sumaba la cuarta comunidad en términos de importancia relativa, el País Vasco, la participación conjunta crecía hasta los dos tercios (62,3\%). Para el año 2005, las tres primeras comunidades habían reforzado su liderazgo al establecerse en un 57,5\% del total de las ventas; pero el País Vasco había sido desplazado por Galicia, que al sumarse a las anteriores representa un $67,2 \%$ de la cuota de mercado".
- Metodología para lograr cada una de los objetivos específicos

- Mediante la realización de eventos como el realizado en Quito del 7 y 8 de Agosto del 2008.

- Conferencias, foros a nivel, oficial, privado y académico.

- Convenios con instituciones como ONGs

- Medios de comunicación

\section{- Resultados}

El desarrollo del proyecto permitió cumplir con la oferta de la entrega de los estudios correspondientes a los diez productos que constituyeron la propuesta.

1. Comercio Justo entre Ecuador y España

2. Manual de Comercio Justo

3. Economía Solidaria

4. Marketing Social

5. Emprendimiento

6. Prospectiva

7. Evento

8. Convenio con la Universidad de Mondragón (España)

9. Sello FLO

10. Empresa de Comercialización 


\section{Conclusiones y Recomendaciones}

\section{Conclusiones}

1. El Comercio Justo como alternativa de comercialización de bienes y servicios tiene un importante despegue en la zona europea y algunos países latinoamericanos como Brasil, México.

2. La modalidad de Comercio Justo para comercializar productos al mercado mundial ha permitido el ingreso de divisas en el último año por \$25 millones, para el Ecuador.

3. No existe apoyo oficial y académico en el Ecuador para favorecer a ésta nueva forma de comercio.

4. A nivel académico la terminología sobre: Comercio Justo, Economía Solidaria, Finanzas Justos, emprendimientos solidarios, es desconocida.

5. La población ecuatoriana desconoce de esta alternativa solidaria para comercializar sus productos, salvo casos excepcionales.

6. El consumidor nacional desconoce esta nueva alternativa de comercio justo, en el Ecuador y los beneficios que esta podría representar para las personas de escasos ingresos.

7. Existen organizaciones privadas, en el Ecuador, que desde hace algunos años están trabajando en este esquema como: Sinchi Sacha, Maquita Cushunchic, Camari, entre otras.

8. Laacademiadesconocedeéstaalternativadecomercialización, salvo la UTE, por el proyecto realizado.

9. Existen una importante red de universidades en el mundo que tienen una oferta académica en Comercio Justo, Economía Solidaria, Finanzas Éticas, entre otros temas.

\section{Recomendaciones}

1. Que la academia incorpore a la malla curricular materias relacionadas con: Comercio Justo, Economía Solidaria, Finanzas Justas, Emprendimientos Solidarios, etc.

2. Hay necesidad de un acercamiento entre las instituciones privadas que están involucradas con comercio justo y solidario y la academia, para desarrollar un atractivo plan para mejorar las condiciones de vida de los menos favorecidos, a través de emprendimientos.

3. Que la Facultad de Ingeniería de Empresas y Negocios de la UTE, tenga como una de las opciones de graduados, una titulación en Economía Solidaría y Comercio Justo.

4. Que se suscriba el convenio macro con la universidad de Mondragón (España), para aprovechar la información y conocimientos en materia de cooperativas productivas y sus resultados exitosos.

5. Que se implemente la propuesta de una empresa Comercializadora en la UTE, en base a uno de los productos identificados en el proyecto de comercio justo.

6. Que se promocione y publicite la UTE, los productos identificados en el proyecto de Comercio Justo.

\section{Bibliografía Consultada}

1. Fundaciónfeda.Org. Directorio de la Dignidad, Ecuador.2008.

2. Lozano, Javier Leopoldo. Diagnóstico de las operaciones de las PYMES, México, 2005.

3. Marcillo, Vaca. Cesar. Economía Solidaria. Ecuador, 2006.

4. Stiglitz, Joseph E. El malestar en la globalización. México, 
2006.

5. Stiglitz, Joseph E. Cómo hacer que funcione la Globalización. Colombia, 2006.

6. Stiglitz, Joseph E. Comercio Justo para todos. México, 2007.

7. Vásquez, Lola. Saltos, Napoleón. Ecuador, su realidad. Ecuador, 2008.

8. Revistas: Varios temas de Comercio Justo, Economía Solidaria facilitados por organizaciones españolas, durante la visita realizada a España en marzo del 2008.

9. Setem. El comercio justo en España 2006. www.setem.org Internet: Artículos sobre Comercio Justo, Economía solidaria, Finanzas solidarias, Micro emprendimientos.

\section{El Investigador}

Experto César Acosta Briones, cacosta@ute.edu.ec

\section{Experto en Comercio Exterior}

e Integración, Escuela Superior

Politécnica del Ejército, 2004

Licenciado en Comunicación Social, Universidad Central del Ecuador, 2005 